\title{
Analysis of local deformations in heterostructures containing short period superlattices by high-resolution transmission electron microscopy
}

\author{
C. Quintana ${ }^{1, a}$, D. Golmayo ${ }^{1}$, M.L. Dotor ${ }^{1}$, and M. Lancin ${ }^{2}$ \\ 1 Instituto de Microelectrónica de Madrid, (IMM-CNM-CSIC) Isaac Newton 8, 28760 Tres Cantos, Madrid, Spain \\ 2 TECSEN, UMR-6122, Fac. Sciences St. Jérome, 13397 Marseille Cedex 20, France
}

Received: 15 April 2003 / Received in final form: 19 September 2003 / Accepted: 7 October 2003 Published online: 17 February 2004 - (c) EDP Sciences

\begin{abstract}
This work describes the application of the Lattice Fringe Spacing Measurement (LFSM) method to the study of complex multiquantum well heterostructures containing both low-misfit and strain compensated short period superlattices in barriers and wells, respectively. $90^{\circ}$-wedge cross-sectional samples have been used. The adequate choice of both experimental conditions and digitized sampling allows the whole heterostructure to be visualized and studied in a single High Resolution Transmission Electron Microscopy (HRTEM) image. Sample preparation and image processing technique are simple and inexpensive, resulting a fast procedure particularly suited for the analysis of large areas. By this way, in a single HRTEM image we have measured, in the growth direction, the lattice spacings at either side of the multiple grown interfaces as well as the period variations of both types of superlattices; in addition, we have measure on the same image the lattice strain in a direction perpendicular to the growth direction by using the LFSM and the Cumulative Sum methods. We have observed local lateral variations within the wells, with regions tensile or compressively strained, while a vestige of the grown SL remains, indicating the occurrence of a strain induced lateral composition modulation process spontaneously produced during the growth of strain compensated short-period superlattices. This is further confirmed in cross-section prepared by the tripod mechanical polisher method.
\end{abstract}

PACS. 68.37.Lp Transmission electron microscopy (TEM) of surfaces, interfaces and thin films including STEM, HRTEM, etc. - 68.65.Cd Superlattices (structure and non-electronic properties) - 81.05.Ea III-V semiconductors: fabrication, treatment, testing and analysis

\section{Introduction}

The present paper is devoted to the characterization by high-resolution transmission electron microscopy (HRTEM) of semiconductor short period superlattices (SL). The use of short period SL is an alternative to the molecular beam epitaxy growth of ternary or quaternary materials of various compositions without the need to include additional source cells or to change the temperatures of cells during growth [1-3]. Both lattice matched and slightly strained pseudoquaternary alloys of the type GaInAsP can be engineered by using $(\mathrm{InP})_{n} /\left(\mathrm{Ga}_{x} \mathrm{In}_{1-x} \mathrm{As}_{y} \mathrm{P}_{1-y}\right)_{m}$ short period SL, the subindex $m, n$ stands for the number of monolayers of each component within a period of the SL. HRTEM is a powerful tool for the study of semiconductors epitaxial layers and SL structures; in particular the control of interfacial strain in the layers that compose a SL is important since

\footnotetext{
${ }^{a}$ e-mail: Carmen.Quintana@curie.u-psud.fr
}

strain can affect the optical and electronic properties of the heterostructure.

Different methods allow for the quantification of HRTEM images and to extract composition and local strain in SL. These methods use procedures dependent $[4,5]$ or independent [6-11] of image contrast interpretation, as those based on the Fourier transform (FT) analysis of HRTEM images [7,8]. The Robertson method [7] is based in the lattice fringe spacing measurements (LFSM) and the calculation of Cumulative Sum (CUSUM) of deviations from an average lattice fringe spacing value [7]. Strain analysis across interfaces by direct measurement of lattice spacings is uncertain when the difference in lattice fringe spacings between the two types of layers at each side of interface is small compared to the scatter of the individual lattice fringe spacing. This is the case of low-misfit systems. The CUSUM method overcomes this limitation when the number of monolayers $m-n$ of each component within a period of the SL is great enough. The method has been used in $\mathrm{InAlSb} / \mathrm{InSb}$ 
bi-layers and SL prepared by the small-angle cleavage technique, providing reliable interface localization and determination of lattice strain. This method has not been used for strain analysis in short period SL ( $m$ and $n$ ranging from 2 to 5 ). For short period SL a great number of pixels per lattice fringe is necessary, limiting the application range of the method to high magnification HRTEM images, digitized with high sampling resolution. Consequently, only a small number of periods of a SL can be studied at a time. Because the lattice fringe spacings depends on experimental conditions $[9,11]$ and of surface relaxation effects [12], the local strain profiles obtained by the LFSM and CUSUM methods can be considered as a first analysis prior to a more accuracy analysis using a method dependent of image contrast.

In this work we have evaluated the application of the LFSM and CUSUM methods to the characterization of a complex multiquantum well laser diode heterostructure, the seven wells made of strain compensated $(\mathrm{InAs})_{2}$ $(\mathrm{GaAs})_{2}$ SL and the six barriers and waveguide made of near lattice-matched $(\mathrm{InP})_{5}\left(\mathrm{Ga}_{x} \mathrm{In}_{1-x} \mathrm{As}\right)_{4}$. The structure was investigated by HRTEM using the $90^{\circ}$-wedge and the tripod mechanical polisher cross-sectional preparations. Lattice distortions were deduced by image processing. The adequate choice of both experimental conditions and digitized sampling allows the whole heterostructure to be visualized and studied in a single HRTEM image. In the growth direction, we have applied the LFSM method to measure the lattice fringe spacing at either side of the multiple grown interfaces, as well as the period variations of both type of superlattices. We have studied the local lateral variations in two directions perpendicular to the growth direction, $\langle 100\rangle$ and [110]. In these directions we have applied the LFSM and the CUSUM method [7]. We have observed local lateral variations with regions tensile or compressively strained indicating the occurrence of a strain induced lateral ordering process. The obtained strain values are of the same order in both types of crosssection preparations.

\section{Experimental}

Samples have been grown at $400{ }^{\circ} \mathrm{C}$ by atomic layer molecular beam epitaxy on (001) InP. The structure is grown without interruptions and using solid sources for arsenic and phosphorus and one single source for each element. The structure consists of: (i) a $100 \mathrm{~nm}$ thick buffer layer of InP; (ii) the waveguide made of $2 \times 34$ periods of near lattice matched $(\operatorname{InP})_{5}\left(\mathrm{Ga}_{x} \operatorname{In}_{1-x} \mathrm{As}\right)_{4}$ SL with $x \approx 0.47$ (hereafter GaInAs) and in the middle of the waveguide (iii) seven quantum well made of strain compensated $\left[7 x(\mathrm{GaAs})_{2}(\mathrm{InAs})_{2}+(\mathrm{GaAs})_{2}\right]$ SL and six barriers of near lattice matched $\left[(\mathrm{GaInAs})_{4}+8 x(\operatorname{InP})_{5}(\mathrm{GaInAs})_{4}\right] \mathrm{SL}$; (iv) a $200 \mathrm{~nm}$ thick cap layer of InP. Nominal thickness of wells and barriers is $8.79 \mathrm{~nm}$ and $22.26 \mathrm{~nm}$ respectively. The total thickness of the heterostructure is $695 \mathrm{~nm}$. The structural and optical quality of the sample was first determined by X-ray diffraction (XRD) and photoluminescence emission at room temperature. The sample has a slightly tensile global strain $(+0.11 \%$ relative to the 004 reflection of the InP substrate and supposing a pseudomorphic structure).

$90^{\circ}$-wedge $\langle 100\rangle$ TEM cross-sectional samples were prepared by cleaving $0.70 \mathrm{~mm}$ square using a scribing apparatus, that provides a good control of sample size, uniform thickness along the [001] direction and good quality of cleaved surfaces [13]. (1-10) TEM cross-sections were prepared using the tripod mechanical polisher method [14] to obtain 1-2 microns thick samples. Afterwards, electron transparence is reached by a few minutes ion thinning using a Precise Ion Polishing System.

Samples were observed at $200 \mathrm{kV}$ or $400 \mathrm{kV}$ using either a Philips CM200 field emission gun TEM, $(C s=$ $1.2 \mathrm{~mm})$ or a JEOL 4000EX $(C s=1.03 \mathrm{~mm})$. A large objective aperture $\left(35 \mathrm{~nm}^{-1}\right.$ or $\left.15 \mathrm{~nm}^{-1}\right)$ was selected to obtain the multi-beam HRTEM images. Magnifications between $300 \mathrm{~K}$ and $800 \mathrm{~K}$ were used.

Processing of the HRTEM image for lattice fringe spacing measurements, includes the following steps:

(1) Image digitalization using a Zeiss PhotoScan scanner with an image sampling resolution of $7-14 \mu \mathrm{m} /$ pixel (3628-1814 pixels/inch).

(2) Image was Fourier-transformed to obtain the digital diffractogram, using CRISP software.

(3) Filtering: the desired reflections are filtered on using smoothed circular masks. The filtered diffractograms were inverse-Fourier-transformed to obtain lattices fringes images from the desired reflections. The fringes near the edges were not included in the analysis [7]. Reflections 002 and 200 were used for the $\langle 100\rangle$ orientation and 002 and 220 reflections for the [1-10] zone axis projection. Figures $1 \mathrm{a}$ and $1 \mathrm{~b}$ show the HRTEM InP image and the corresponding 002 lattice fringe image.

(4) For the LFSM and CUSUM methods, we have implemented a Mat-Lab program as follow: for an image of $M \times N$ pixels (rows $\times$ columns) formed by a set of $K$ vertical (or horizontal) fringes ( $K-1$ spacings), the position of the centres of successive fringes is calculated in each row (or column) using the $n$-pixels interpolation Mat-lab Spline function. We have used $n=10$ pixels for our calculations. Figure 1c displays an example of the polynomial fit interpolation applied to the raw pixel intensity data. The maxima of the interpolated function are taken as the centres of the lattice fringes. The positions of these $M(N) \times(K-1)$ maxima are calculated and stocked in a matrice-file. The spacing between two successive maxima in the $i$ row (or column) is denoted $Z_{i}$ and expressed in pixels. The average fringe spacing $Z_{K}$, the average fringe spacing across each row (or column) $Z_{K}^{\prime}$ and the total average of fringe spacing $Z_{T}$, are then calculated. The $h k l$-LFSM plot displays the calculated $h \mathrm{kl}-Z_{K}$ versus the number of spacing $(K-1)$.

(5) Interface localization and determination of lattice strain: the CUSUM plot is obtained by computing and plotting the $\Sigma\left(Z_{K}-Z_{T}\right)$ versus number of spacing $(K-1)$ or versus $\Sigma Z_{K}$. Linear regions in the CUSUM 

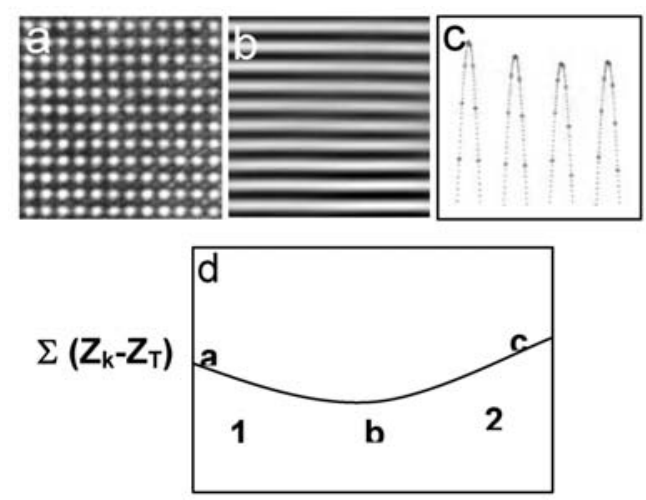

$\Sigma\left(\mathbf{Z}_{\mathrm{k}}\right)$

Fig. 1. (a) and (b) show the HRTEM InP image and the corresponding 002 lattice fringe image; (c) displays an example of the 10 pixels polynomial fit interpolation applied to the raw pixel intensity data; (d) displays the CUSUM plot; slope discontinuities indicate zones where fringe spacing has changed.

plot (Fig. 1d) represent zones where the fringe spacing is constant and vortices indicate zones where the fringe spacing has changed. This representation allows for an easier visualization of the interfaces between two sets of fringes of different spacing and for computing lattice strain across the interfaces [7]. Strain is calculated from $e=m_{2}-m_{1} / 1-m_{2}$, where $e$ is the misfit perpendicular to the interface in terms of the slopes $m_{i}$ of two consecutive layers, and $m=\left(Z_{K}-Z_{T}\right) / \Sigma Z_{K}$ is the slope of a layer in the cumulative sum plot.

(6) When the number of layers at the interface sides is small (2-5 monolayers), the CUSUM method provides good results only if the number of pixels per fringe spacing is high. We have shown this by simulating the lattice fringe image of an interface with lattice spacings of 12 and 11.88 pixels (difference of $1 \%$ at each side). Noise has been added to the sine function that generates the fringes. Figure 2 shows the corresponding CUSUM plot, where the interface position is unclear. Increasing the number of pixels (34 and 33.66, the same difference of $1 \%$ ), the interface is clearly shown in the CUSUM plot. This shows that only a small number of periods of a SL can be studied at a time.

In the case of HRTEM images containing several periods of a short period SL, satellite reflections are present in the digital diffractogram around the principal 002 reflection. The spatial resolution of the filtered image depends directly on the mask radius; a mask smaller than the SL period will average the fringe spacings over several periods, but when the mask includes the SL spots the $(002+S L)-L F S M$ plot reproduces local differences of the [002] lattice parameter of each SL layer, if the difference in the lattice fringe spacing is large compared to the scatter of the individual lattice fringe spacing. We have shown this by simulating the 002 lattice fringe image corresponding to a short period SL formed by $a=5$ and $b=4$ monolayers of spacing $d_{a}=12$ and $d_{b}=11$ pixels respectively, and including growth irregularities on the $(a+b)$ period.

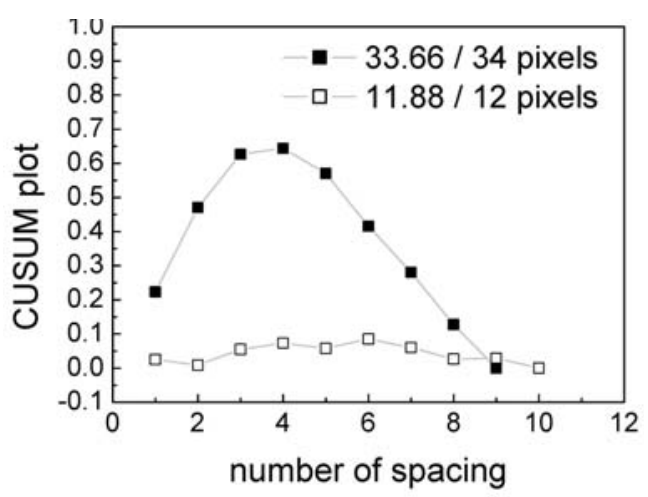

Fig. 2. CUSUM plots corresponding to two different simulated interface, with lattice spacing at each side of the interface of 12 and 11.88 pixels for the first one, and 34 and 33.66 pixels for the second one.

Figure 3a shows five periods of the simulated lattice fringe image. The simulated sequence is: $\left(5^{\star} d_{a}+4^{\star} d_{b}\right)+\left(4^{\star} d_{a}+\right.$ $\left.6^{\star} d_{b}\right)+\left(5^{\star} d_{a}+4^{\star} d_{b}\right)+\left(4^{\star} d_{a}+6^{\star} d_{b}\right)+\left(5^{\star} d_{a}+4^{\star} d_{b}\right)$. Gaussian noise (mean value $=0$, variance $=0.001$ ) has been added to the sine function that generates the fringes. The inset shows a principal reflection and the SL satellites around it, in the corresponding digital diffractogram. Circles indicate the masks used to filter on the reflections, the small one to filter on the principal, and the large one for the principal plus SL satellite reflections. Figure 3b shows the 002-FLSM plot of the simulated lattice fringe image, a square periodic function with maximal and minimal values of 12 and 11 pixels, respectively. Figure 3c shows the 002LFSM plot derived from the inverse Fourier transform of the simulated lattice fringe image obtained using the small mask to filter on the principal reflection, corresponding to $1 / d_{a}$ and $1 / d_{b}$ spatial frequencies. The plot shows a continuum function, as corresponds to an averaged value. The 002-CUSUM plot is also shown in this figure; it does not give additional information on the spacing differences nor on the position of interfaces. The 002-LFSM plot shown in Figure 3d was derived from the inverse Fourier transform image obtained using the large mask to filter on the principal reflection plus the SL satellite reflections. Now, the plot displays a periodic function with maximal and minimal mean values of $11.98(0.06)$ and 11.05 (0.05) close to the simulated values, 12 and 11 pixels, and the period of the simulated sequence; the quantities in brackets denote one standard deviation error limits.

Choice of a micrograph for its study: among a set of HRTEM micrographs obtained in different experimental conditions on a set of $90^{\circ}$-wedge sample preparations, we have chosen the micrograph shown in Figure 4 according to the following criteria:

(1) A value of magnification $(M=300 \mathrm{~K})$ that allow us to visualize most of the heterostructure: part of the InP buffer (that constitutes our internal standard), the bottom waveguide, 4 well and 4 barriers.

(2) A well oriented $\langle 100\rangle$ sample, to obtain symmetrical $\{200\}$ reflections.

(3) A defocus that allows a good contrast of the SL. 

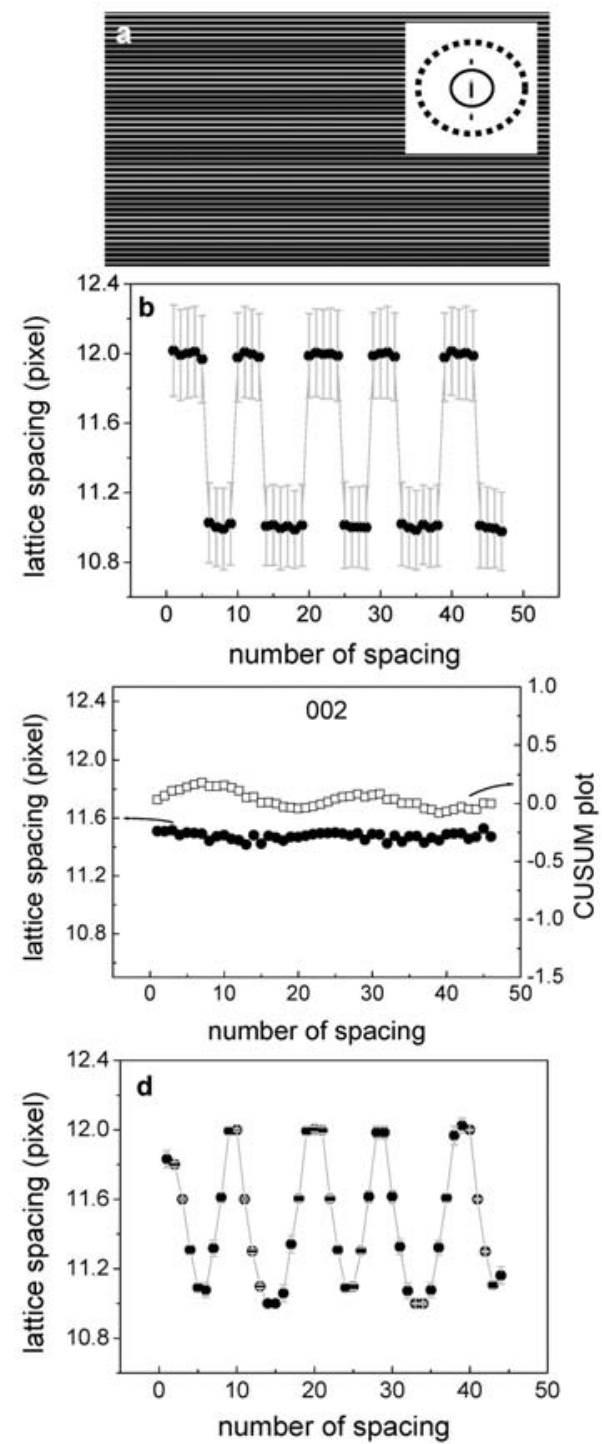

Fig. 3. (a) shows five periods of a simulated lattice fringe image. The inset shows a part of the corresponding digital diffractogram. Circles indicate the masks used to filter on the reflections, the principal (small mask) or the (principal + SL satellite) reflections; (b) shows the 002 FLSM plot of the simulated lattice fringe image, a square periodic function with maximal and minimal values of 12 and 11 pixels, respectively; (c) shows the 002-LFSM plot derived from the inverse Fourier transform of the simulated lattice fringe image obtained using the small mask. The 002-CUSUM plot is also shown in this figure; (d) shows The 002-LFSM plot was derived from the inverse Fourier transform image obtained using the large mask.

(4) A digitalization at high resolution $(7 \mu \mathrm{m} /$ pixel). In these conditions the resolution of numerical image is about $0.024 \mathrm{~nm} /$ pixels and the number of pixels per 002 lattice spacing about 12. In the corresponding $1024 \times 1024$ pixels FT, the module of the reciprocal vector $g_{002}$ is 84 pixels.

Accuracy of the 002-LFSM on the $90^{\circ}$-wedge crosssectional samples: as thickness of a $90^{\circ}$-wedge preparation changes continuously in the in plane directions, we have

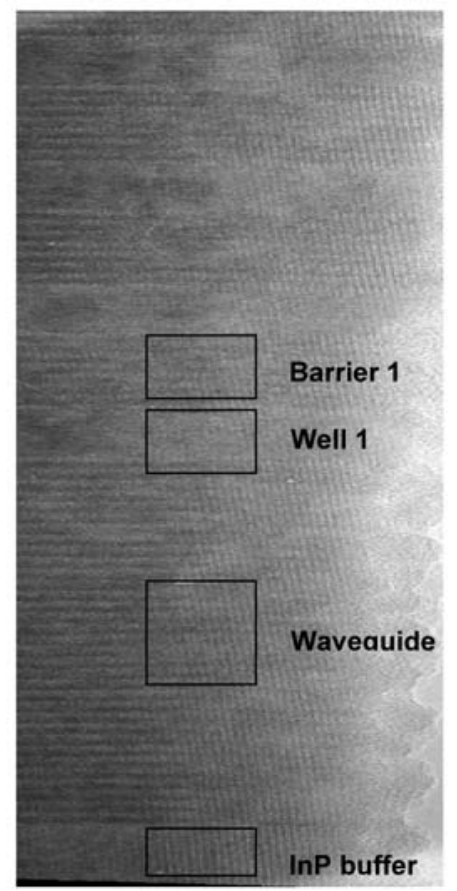

Fig. 4. $90^{\circ}$-wedge $\langle 100\rangle$ cross-section HRTEM image, indicating the analyzed zones. The image resolution is $0.024 \mathrm{~nm} /$ pixel for a microscopic magnification of $300 \mathrm{~K}$.

tested its effects in a zone of the InP buffer (see Fig. 4), taken as a perfect crystal and including 40 unit cells with a thickness variation of $24 \mathrm{~nm}$, ranging from $\approx 2 \mathrm{~nm}$ to $\approx 26 \mathrm{~nm}$. A set of 002 lattice fringe images are obtained by changing the mask filter radius $(5,12,22$ and 42 pixels). Results are shown in Figure 5. A 5 pixels radius only includes the low frequencies allowing the long distance lateral variations to be observed, while a 22 pixels radius includes the satellite reflections of the short period SL in the well $\left(g_{002} / 4\right)$. Figures 5 a to $5 \mathrm{c}$ show the 002 lattice fringe images, which appear distorted for the larger mask radius. In Figures $5 \mathrm{~d}$ to $5 \mathrm{f}$ there are shown the corresponding plots of the average fringe spacing $002-Z_{K}$ versus the number of spacing. Here, measurements average the possible lattice spacing variations due to thickness variation. Increasing radius mask does not change the averaged fringe spacing $Z_{K}$, but increases standard deviation and distorts the fringes at the borders. For a radius of 22 pixels, and eliminating two fringes at each border, the relative standard deviation of the average fringe spacing is about $0.2 \%$. In Figures $5 \mathrm{~g}$ to $5 \mathrm{i}$ there are shown the corresponding plots of the average fringe spacing across each column 002- $Z_{K}^{\prime}$ versus the number of column, from thick to thin regions; variation in lattice spacing between thick and thin regions are always smaller than $0.2 \%$, showing a small effect of the $24 \mathrm{~nm}$ thickness variation on the 002 lattice spacings measurements. According to these results, we have used a 22 pixels mask for measurements on the SLs in the growth direction, and a 5 pixels mask for reference measurements on InP buffer and for the 200 and 220 direction measurements on the SLs. 

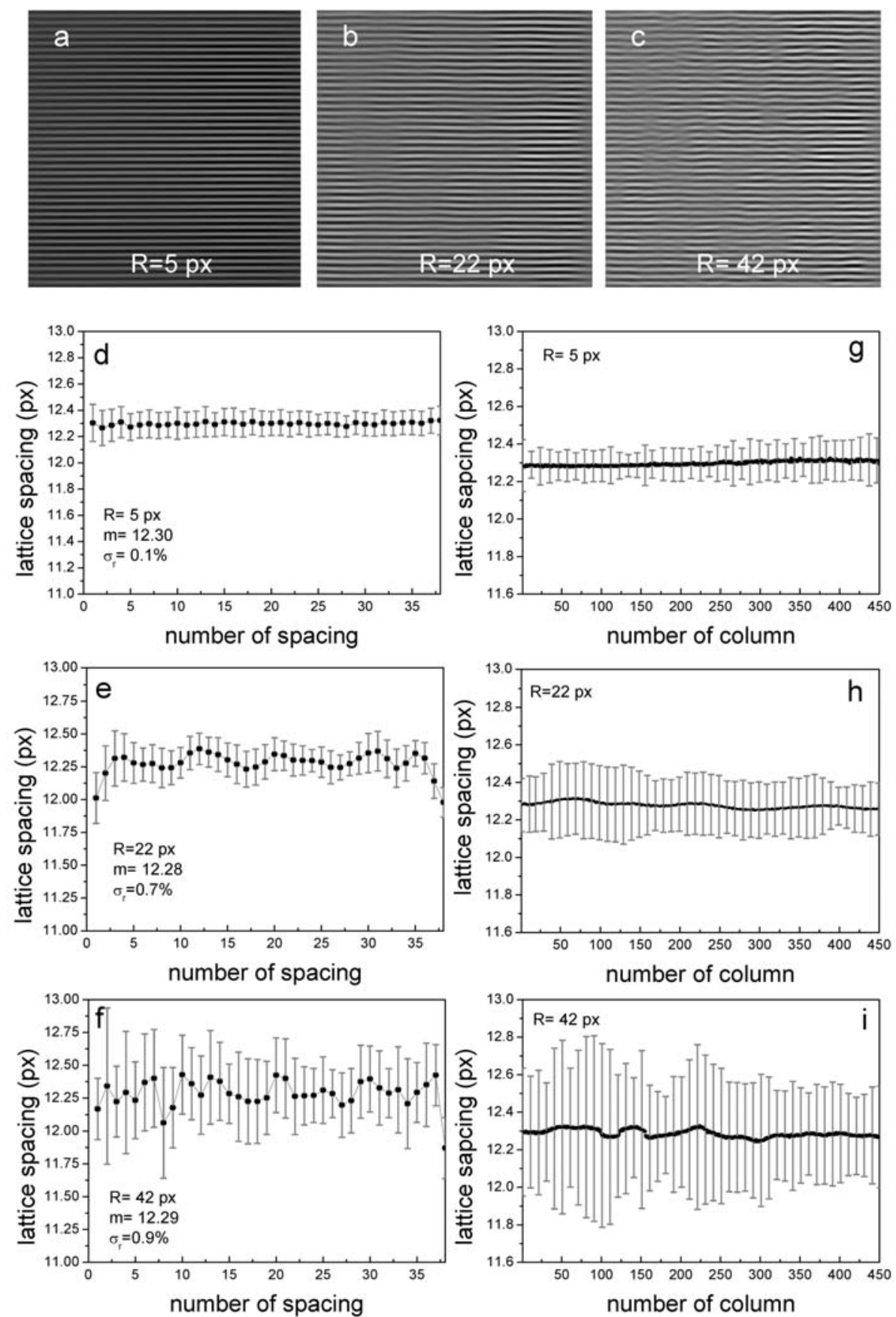

Fig. 5. (a, c) set of 002 lattice fringe images of the selected region of Figure 4 corresponding to the InP buffer, obtained for mask filter radius of $R=5,22$ and 42 pixels respectively; (e, f) show the corresponding plots of the average fringe spacings, $Z_{K}$ versus number of spacing; mean and standard deviation are shown; (g, i) show the corresponding plots of the average fringe spacings across each column, $Z_{K}^{\prime}$ versus number of column.

Internal calibration standard: the InP fringe spacing of the buffer is used as internal calibration standard. Figure 6a shows the selected region of Figure 4 corresponding to the InP buffer. Figure $6 \mathrm{~b}$ shows the hkl-LFSM plots for the 002 and 200 lattice fringe images. The averaged 002 and 200 fringe spacing is $12.30(0.012)$ and $12.25(0.015)$ pixels respectively. Taking the [001] direction as reference $\left(d_{002}=0.2934 \mathrm{~nm}\right)$ the experimental image resolution is
$0.0238 \mathrm{~nm} /$ pixels. That gives a value of $0.292 \mathrm{~nm}$ for the in plan $d_{200}$ spacing, a difference of about $0.4 \%$ relative to $d_{002}$. Furthermore, the angle formed by directions [001] and [100] is $\alpha=90.9^{\circ} \pm 0.1$. We attribute this deformation of the InP to a defect of the TEM images at mean magnification. In Figure $4, \alpha=90.8^{\circ} \pm 0.1$ in the bottom and top of the image and $90.2^{\circ} \pm 0.1$ in the center. In images at higher magnification, this deformation is not observed. 

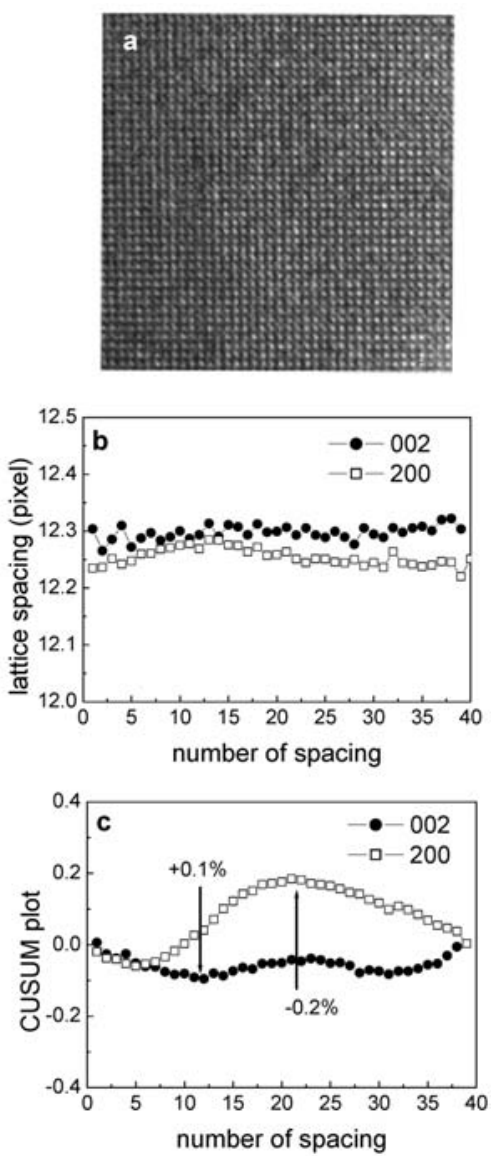

Fig. 6. (a) shows the selected region of Figure 4 corresponding to the InP buffer; (b) shows the hkl-LFSM plots for the 002 and 200 lattice fringe images, using a 5 pixels mask; (c) shows the CUSUM plots for the 002 and 200 lattice fringe image.

The CUSUM plots for the 002 and 200 lattice fringe image are shown in Figure 6c. Slope changes can be due to defects derived from sample preparation, TEM observation and/or image processing, and allow us to evaluate the residual or minimal strain we can measure in our HRTEM image. These minimal strains are $0.1 \%$ in the [001] direction and $0.2 \%$ in the [100] direction. Then, we consider $0.2 \%$ as the smaller limit for strain measurements.

\section{Results}

We have carried out the study of lattice spacing variations in the above cited short period SL heterostructure. The films are coherent to the InP substrate, as evidenced both by XRD and TEM. Lattice spacing has been measured both in the growth direction [001] (hereafter out of the plane direction) as well as in two in the plane directions, $\langle 100\rangle$ and [110], perpendicular to the [001] growth direction. For measurements in out of the plane direction we have used the above described LFSM method on the $\langle 100\rangle$ cross-sectional samples, while for measurements in the plane directions we have used the LFSM and CUSUM method.
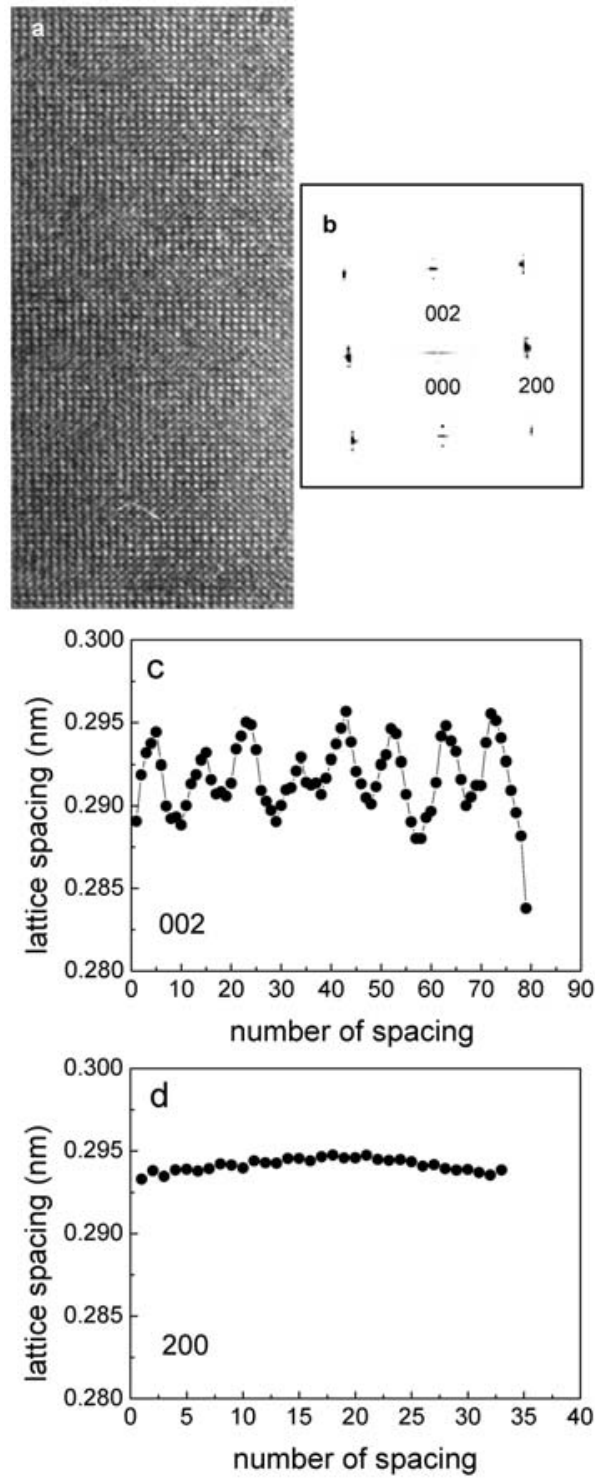

Fig. 7. (a) displays eight periods of the $(\mathrm{GaInAs})_{4}(\mathrm{InP})_{5} \mathrm{SL}$ of Figure 4 waveguide; (b) the digital diffractogram shows the SL satellites; (c, d) show the (002)-LFSM and the (200)-LFSM plots, respectively.

\subsection{Out of the plane spacing measurements}

Figure 7 a displays an $914 \times 414$ pixel $(\approx 22 \mathrm{~nm} \times 10 \mathrm{~nm})$ subset of the original $1024 \times 512$ pixel image corresponding to 8 periods of the near lattice matched $(\mathrm{GaInAs})_{4}(\mathrm{InP})_{5}$ SL in the middle of the bottom waveguide (see Fig. 4). The digital diffractogram of Figure $7 \mathrm{~b}$ shows the SL satellites. Figure 7c shows the 002-LFSM plot where a periodic modulation of the spacing can be observed with an averaged period of 9.5 (0.9) monolayers, close to the nominal period of the SL. Values of averaged maximal and minimal spacing are $0.294(0.002) \mathrm{nm}$ and $0.289(0.002) \mathrm{nm}$.

Figure 8a displays an $461 \times 432$ pixel $(\approx 11 \mathrm{~nm} \times 10 \mathrm{~nm})$ image of the selected region of Figure 4 corresponding to the first well of the strain compensated $(\mathrm{GaAs})_{2}(\mathrm{InAs})_{2}$ 

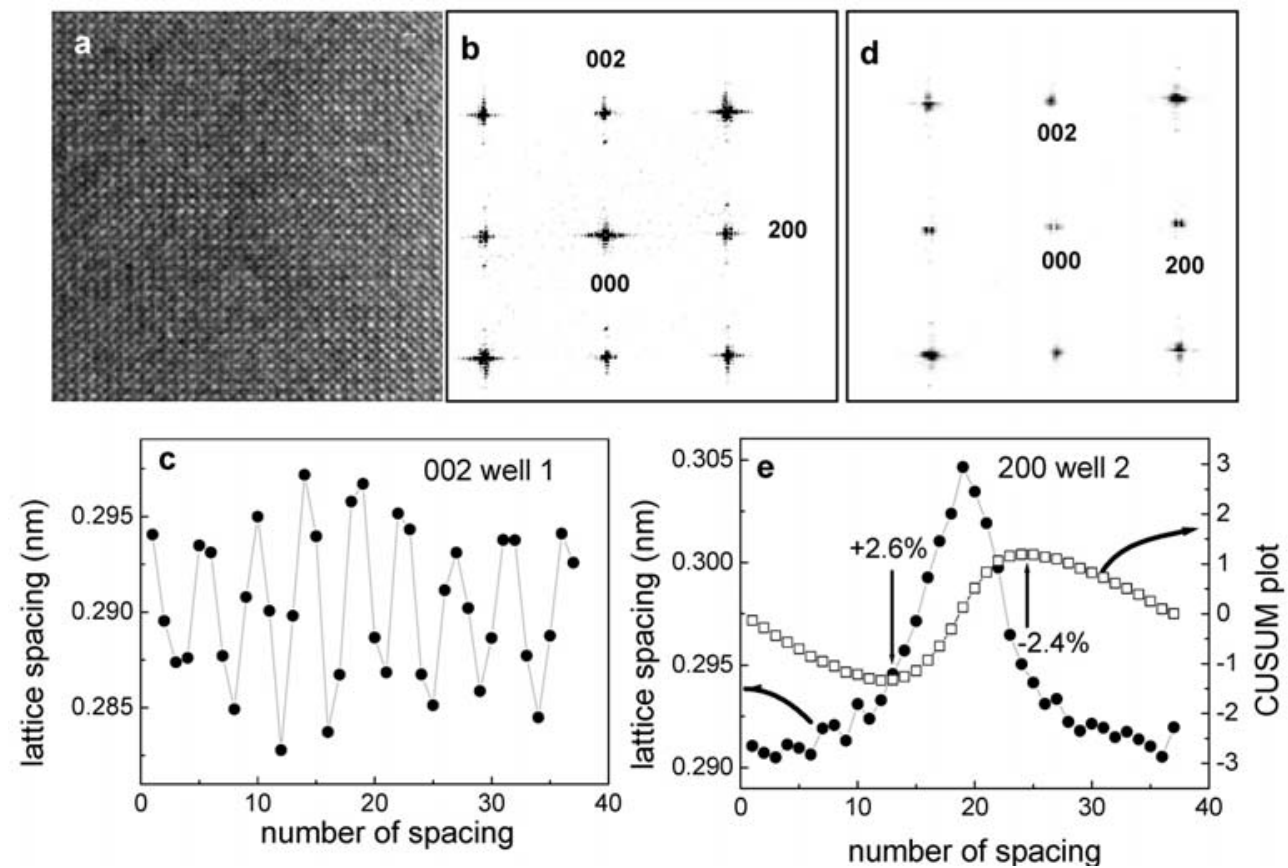

Fig. 8. (a) displays the selected region of Figure 4 corresponding to the first well of the heterostructure; (b) shows the digital diffractogram, where the SL satellites can be observed in the [001] direction and the 200 reflection appears elongated; (c) displays the (002)-LFSM plot; (d) shows the digital diffractogram corresponding to the second well, where a double 200 reflection appears; (e) displays the second well 200-LFSM plot and the corresponding 200-CUSUM plot.

short period SL. Figure 8b shows the digital diffractogram, where the SL satellites can be observed in the [001] direction; Figure 8c displays the 002-LFSM plot, showing a periodic function of averaged period of $4.4(0.6)$ monolayers, averaged lattice spacing of $0.290(0.004) \mathrm{nm}$ and averaged maximal and minimal spacing of $0.295(0.005) \mathrm{nm}$ and $0.286(0.005) \mathrm{nm}$ respectively.

Figures 9a and 9b show image and digital diffractogram from the selected region of Figure 4 corresponding to the first $(\mathrm{GaInAs})_{4}(\mathrm{InP})_{5}$ SL barrier, of dimensions $1000 \times 424$ pixels $(\approx 24 \mathrm{~nm} \times 10 \mathrm{~nm})$. As in the waveguide region, periodic lattice spacing variations can be observed in the 002-LFSM plot of Figure 9c, with averaged period of 9.2 (1.0) monolayers, averaged lattice spacing of 0.290 $(0.003) \mathrm{nm}$ and averaged maximal and minimal spacing of $0.292(0.002) \mathrm{nm}$ and $0.288(0.002) \mathrm{nm}$.

The results show that the 002-LFSM plot reproduces the local spacing differences across the interface of low-misfit and strain compensated short period SL, giving local information on the out of the plane direction of large area of complex structures with multiple interfaces as those used in multiquantum well laser diode heterostructures. In the set of measurements made in Figure 4, the whole waveguide and fourth barriers made of $(\mathrm{InP})_{5}(\mathrm{GaInAs})_{4} \mathrm{SL}$, we have found a periodic spacing modulation with a global averaged period of 9.4 (0.1) monolayers and a global averaged lattice spacing of $0.291(0.001) \mathrm{nm}$ varying from 0.294 (0.001) nm to 0.290 $(0.001) \mathrm{nm}$. In the measurements made on the first fourth wells made of $(\mathrm{GaAs})_{2}(\mathrm{InAs})_{2} \mathrm{SL}$, we have also found a periodic spacing modulation with a global averaged period of $4.12(0.25)$ monolayers, a global averaged lattice spacing of $0.290(0.001) \mathrm{nm}$, varying from $0.294(0.001) \mathrm{nm}$ to $0.285(0.001) \mathrm{nm}$.

\subsection{In the plane spacing measurements}

For measurements in the plane directions we have used the CUSUM method on the $90^{\circ}$-wedge $\langle 100\rangle$ (Fig. 4) and the tripod mechanical polished [110] (Fig. 10) cross-sectional samples. We present first the analysis of the $90^{\circ}$-wedge sample.

In the waveguide (see Fig. 7d), no local lattice variations are observed in the near lattice matched $(\mathrm{InP})_{5}(\mathrm{GaInAs})_{4} \mathrm{SL}$; strain in the 200-LFSM plot is similar to the residual value found in $\mathrm{InP}(0.2 \%)$.

In the digital diffractogram of the (GaAs) $)_{2}(\mathrm{InAs})_{2} \mathrm{SL}$ of the wells, the 200 reflection appears elongated (i.e. first well, Fig. 8b) or clearly separated in two spots (i.e. second well, Fig. 8d). In this second well, the corresponding 200 spacing values are $0.298(0.005) \mathrm{nm}$ and $0.283(0.005) \mathrm{nm}$. Figure 8e displays the second well 200-LFSM and the corresponding CUSUM plots, showing the spatial localization of the 200 lattice parameter variations; the central region of the image has the largest lattice spacing, and the calculated strains relative to the average lattice parameter $0.294(0.004) \mathrm{nm}$ are $+2.6 \%$ and $-2.4 \%$. Strain value depends on the analyzed region; the higher value we have found corresponds to thesecond well. 

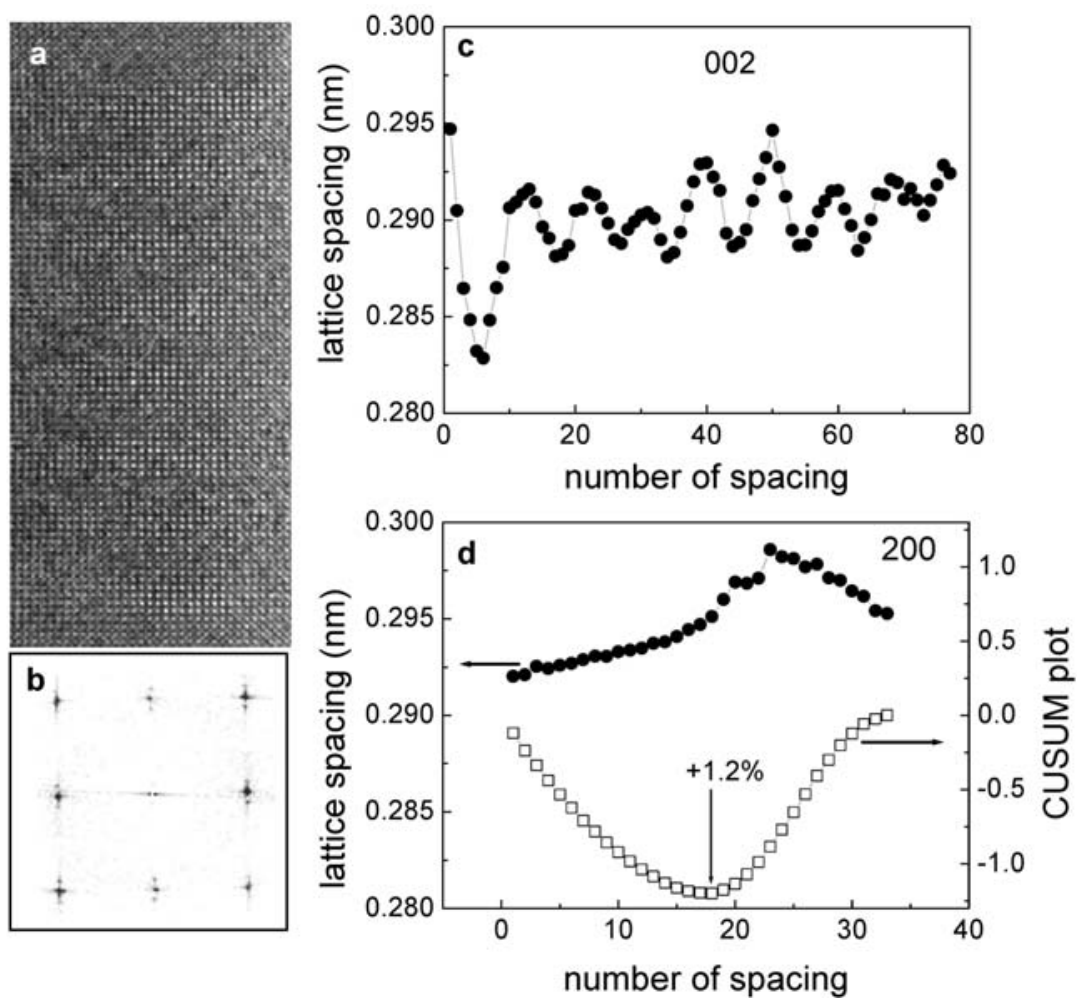

Fig. 9. (a) shows the selected region of Figure 4 corresponding to the first barrier; (b) shows the digital diffractogram, where the SL satellites can be observed in the [001] direction; (c) shows the (002)-LFSM plot; (d) shows the 200-LFSM plot and the corresponding CUSUM plot.

In the first barrier (Fig. 9d) there is a deformation of $+1.2 \%$ in the in plan [100] direction similar to that observed in the first well region (not shown).

Due to sample thickness variation in the $\langle 100\rangle$ direction, it is not possible to study lateral variations in a large distance in the $90^{\circ}$-wedge preparations. For this reason we have studied the local variations of the 220 lattice parameter on the HRTEM images obtained from the tripod mechanical polished [110] (Fig. 10) cross-sections. The images were acquired at a magnification of $800 \mathrm{~K}$ and with a high image sampling resolution of $0.0085 \mathrm{~nm} /$ pixel (34.5 pixels by 002 fringe spacing and 24.4 pixels per 220 fringe spacing).

The HRTEM image of Figure 10a shows a well within two barriers and the analyzed zones labeled 1-3. Dimensions of each analyzed area are $1024 \times 1024$ pixels $(\approx 9 \mathrm{~nm} \times 9 \mathrm{~nm})$. Figure 10b shows the 220-LFSM plot, from zone 1 to zone 3 . In the central region (zone 2) the spacing $0.2079(0.0005)$ is practically constant and close to the $220 \mathrm{InP}$ lattice parameter $(0.2075 \mathrm{~nm})$. But in the adjacent regions ( 1 and 3 ) the 220 lattice spacing presents variations. Figure 10c shows the corresponding CUSUM plot and the calculated values of strain of $-1.8 \%,+0.4 \%$ and $-2.4 \%$. These values are of the same order of those found in the 200 direction on the $90^{\circ}$-wedge preparations, showing that the $90^{\circ}$-wedge preparation can be used for in plane deformation measurements.

\section{Discussion}

Sample preparation and image processing technique are simple and inexpensive, resulting a fast procedure particularly suited for a first analysis of large areas prior to a more accurate analysis using a method dependent of image contrast. $90^{\circ}$-wedge cross-sectional samples have been used for analysis of local deformations in heterostructures containing short period SL. Thickness in the [001] direction is constant, but varies rapidly in the $\langle 100\rangle$ direction, limiting the analysis to regions of about 40 unit cells; by the contrary, an advantage of the method is that the $1500 \mathrm{~nm}$ thick substrate helps minimizing relaxation of the grown structure during TEM sample preparation, and serves as internal reference. The adequate choice of both experimental conditions and digitized sampling allows the whole heterostructure to be visualized and studied in a single HRTEM image.

The 002-LFSM plots reproduces the local spacing differences across the interface in the growth direction of low-misfit and strain compensated short period SL. Averaged 002 lattice spacing indicates that the overall $90^{\circ}$ wedge sample (thin sample) is slightly tensile strained, in agreement to XRD measurements (thick sample), indicating that TEM sample is only weakly, if at all, relaxed [12]. Variations of lattice spacing and periods relative to the nominally grown values can be partially due to the growth control limits. During atomic layer molecular beam epitaxy of the $(\mathrm{InP})_{5}(\mathrm{GaInAs})_{4} \mathrm{SL}, \mathrm{P}_{2}$ and $\mathrm{As}_{4}$ are 

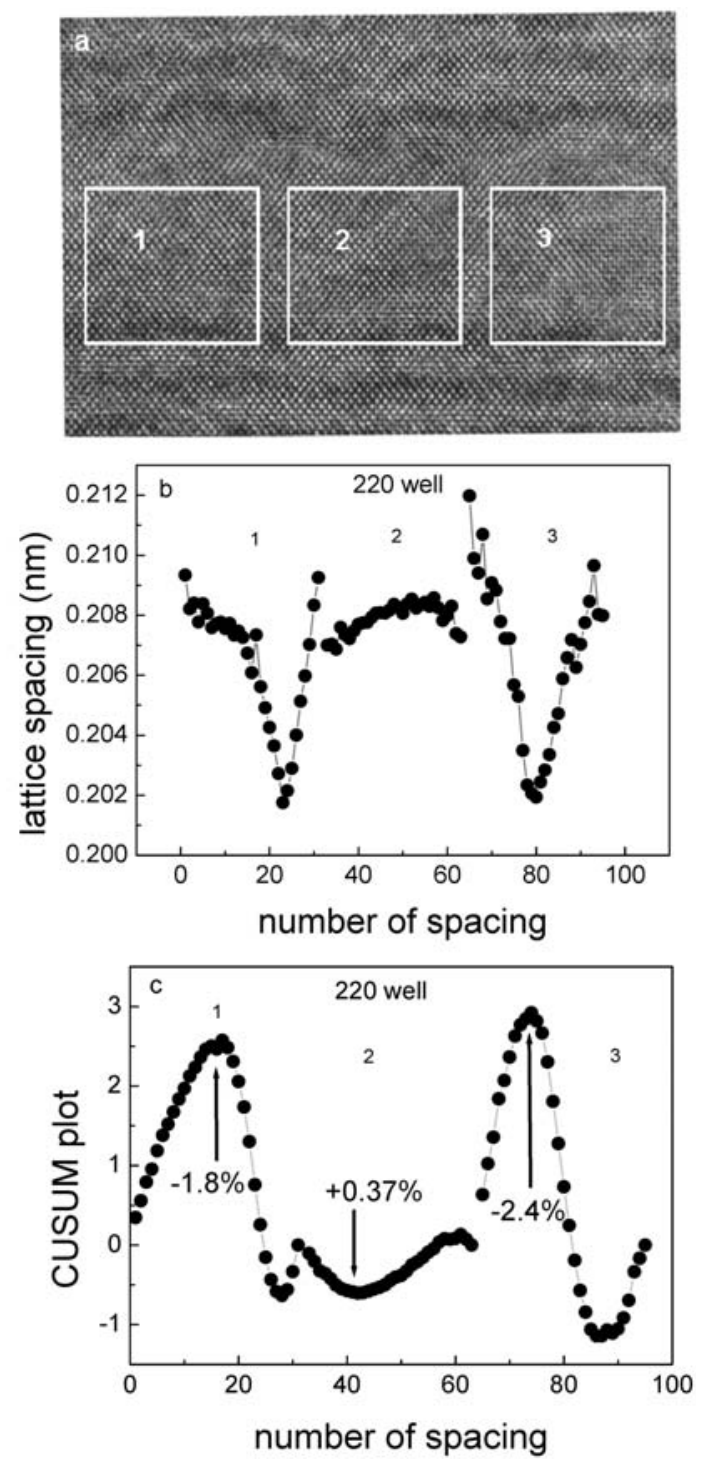

Fig. 10. (a) shows a well within two barriers and the analyzed zones labelled 1-3, corresponding to the tripod mechanical polished $\langle 110\rangle$ cross-section image; (b) shows the 220-LFSM plot, from zone 1 to zone 3 ; (c) shows the corresponding CUSUM plot and the calculated values of strain. Microscopic magnification was $800 \mathrm{~K}$, and image resolution $0.085 \mathrm{~nm} /$ pixel.

supplied separately in successive pulses to group III terminated intermediate surfaces, avoiding competition. However, residual group $\mathrm{V}$ flux during the intervals between group $\mathrm{V}$ pulses sets a limit to the purity of the individual layers [15]. During the (GaAs) $)_{2}(\mathrm{InAs})_{2}$ growth, only one element $\mathrm{V}\left(\mathrm{As}_{4}\right)$ is supplied in successive pulses to alternated group III terminated intermediate surfaces (Ga or In). The transients in the group III shutters set a limit to the purity of the individual GaAs and InAs layers. Finally, the control of ternary composition and number of monolayers of the superlattice components is limited by the accuracy of element III evaporation rate control.

We have found local variations of the 200 lattice spacings only in the strain compensated short period
$(\mathrm{GaAs})_{2}(\mathrm{InAs})_{2}$ SL of the wells (Fig. 8), the large lattice spacing corresponding to an In-rich zone surrounded by regions of lower In content; we have found that this deformation is partially transmitted to the barrier from the immediate well. Not lateral variations have been founded in the buffer and waveguide. In addition, in the thinned preparation (Fig. 10) (thickness about $50 \mathrm{~nm}$ ) we have found in the wells lateral variations of the 220 lattice spacing; here the analyzed zone shows the existence of two Ga-rich region, separated by about $10 \mathrm{~nm}$. This lateral spacing variation can be attributed to the occurrence of lateral composition modulation produced spontaneously during the growth of strain compensated short-period SL consisting of alternating pairs of layers whose relaxed lattice constants lie on either side the lattice constant of the host crystal [16-18]. During growth, the film composition spontaneously modulates in a direction perpendicular to both the [001] growth direction and the [1-10] direction. This orientation is believed to result from fast diffusion along dimmer in the [1-10] direction, forming extended sheets of alternatively In-rich and Ga-rich material [18]. The observed results on local lateral variations of the 200 and 220 lattice parameters, with regions tensile or compressively strained, indicates the occurrence of a strain induced lateral composition modulation process, while vestige of the grown strain compensated short period SL remains [19].

\section{Conclusions}

We have applied the LFSM method to study a multiquantum well heterostructure containing both low-misfit and strain compensated short period SLs. The method can be applied to the fast and simple $90^{\circ}$-wedge cross-sectional preparations, and the processing of digital images can be easily implemented in a PC. The adequate choice of both experimental conditions and digitized sampling allows the whole heterostructure to be visualized and studied in a single HRTEM image, resulting a fast procedure particularly suited for the analysis of large areas. The 002-LFSM plots reproduces the local spacing differences across the interface in the growth direction, founding that the overall sample is slightly tensile strained, in agreement to XRD measurements. On the same image, we have measure the 200 lattice parameter by using the LFSM and the CUSUM methods to obtain local lateral variations within the wells, with regions tensile or compressively strained, while a vestige of the grown SL remains, indicating the occurrence of a strain induced lateral composition modulation process produced spontaneously during growth. This is further confirmed in cross-section prepared by the tripod mechanical polisher method, where local lateral variations of the 220 lattice parameter are observed. The local strain profiles can be used as input data for a more accuracy analysis with methods depending of image contrast.

Financial support from the Spanish Commission of Science and Technology (project TIC99-0645-C05-05) and Ministry of Education and Culture (M. Lancin) are gratefully acknowledged. 
The authors express their sincere thanks to A. Guerrero for his support in the CUSUM program, to J. Thibault who invited them to take benefit of the $4000 \mathrm{EX}$ microscope available in the CEN-Grenoble, and to A. Sanz-Hervás, from Depto. Tecnología Electrónica, E.T.S.I.T.-U.P.M., Madrid, for his support in XRD measurements.

\section{References}

1. J.D. Song, J.S. Yu, J.M. Kim, S.J. Bae, Y.T. Lee, Appl. Phys. Lett. 80, 4650 (2002)

2. I.J. Fritz, M.J. Hafich, J.F. Klem, S.A. Casalnuovo, Electron. Lett. 35, 171 (1999)

3. P.A. Postigo, D. Golmayo, H. Gómez, D. Rodríguez, M.L. Dotor, Jpn. J. Appl. Phys. 41, L565 (2002)

4. A. Ourmazd, F.H. Baumann, M. Bode, Y. Kim, Ultramicroscopy 34, 237 (1990)

5. M. Lamy, J. Thibault, Ultramicroscopy 84, 101 (2000).

6. R. Bierwolf, M. Hohenstein, F. Phillipp, O. Brandt, G.E. Crook, K. Ploog, Ultramicroscopy 49, 273 (1993)

7. M.D. Robertson, J.E. Currie, J.M. Corbett, J.B. Webb, Ultramicroscopy 58, 175 (1995)
8. M.J. Hÿtch, E. Snoeck, R. Kilaas, Ultramicroscopy 74, 131 (1998)

9. M.J. Hÿtch, T. Plamann, Ultramicroscopy 87, 199 (2001)

10. P.H. Jouneau, A. Tardot, G. Feuillet, H. Mariette, J. Cibert, J. Appl. Phys. 75 ,7310 (1994)

11. K. Tillmann, M. Lentzen, R. Rosenfeld, Ultramicroscopy 83, $111(2000)$

12. M.M.J. Treacy, J.M. Gibson, A. Howie, Philos. Mag. A 51, 389 (1985)

13. T. Boone, S. Nakahara, Mater. Res. Soc. Symp. Proc. 115, $81(1988)$

14. J. Benedict, R. Anderson, S.J. Klepeis, Mater. Res. Soc. Symp. Proc. 199, 189 (1990)

15. M.L. Dotor, P. Huertas, D. Golmayo, F. Briones, Appl. Phys. Lett. 62, 891 (1993)

16. K.C. Hsieh, J.N. Baillargeon, K.Y. Cheng, Appl. Phys. Lett. 57, 2244 (1990)

17. D. Golmayo, M.L. Dotor, C. Quintana, J. Cryst. Growth 253, 167 (2003)

18. R.D. Twesten, D.M. Follstaedt, S.R. Lee, E.D. Jones, J.L. Reno, J.M. Millunchick, A.G. Norman, S.P. Ahrenkiel, A. Mascarenhas, Phys. Rev. B 60, 13619 (1999)

19. Y. Zhang, A. Mascarenhas, Phys. Rev. B 57, 12245 (1998) 